\title{
Visions of the Uterine Cavity
}

ISSN: 2689-2707

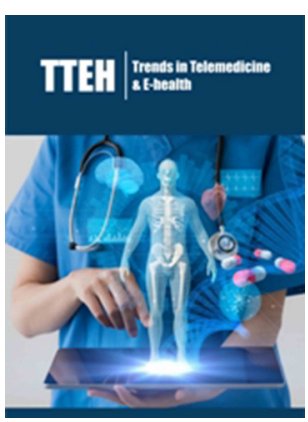

*Corresponding author: Liselotte Mettler, German Medical Center (GMC), Dubai Health Care City (DHCC), Dubai, UAE

Submission: 僵 January 30, 2020

Published: 㑑 March 12, 2020

Volume 2 - Issue 3

How to cite this article: Liselotte Mettler, Amaya Carreras Ugarte, Anupama Deenadayal Mettler, Wael Sammur, Meenu Agarwal, Ibrahim Alkatout. Visions of the Uterine Cavity. Trends Telemed E-Health 2(3). TTEH. 000537. 2020. DOI: 10.31031/TTEH.2020.02.000537

Copyright@ Liselotte M, This article is distributed under the terms of the Creative Commons Attribution 4.0 International License, which permits unrestricted use and redistribution provided that the original author and source are credited.

\author{
Liselotte Mettler ${ }^{1,2 *}$, Amaya Carreras Ugarte ${ }^{1}$, Anupama Deenadayal Mettler ${ }^{2}$, \\ Wael Sammur ${ }^{3}$, Meenu Agarwal ${ }^{4}$ and Ibrahim Alkatout ${ }^{2}$ \\ ${ }^{1}$ German Medical Center (GMC), Dubai Health Care City (DHCC), Dubai, UAE \\ ${ }^{2}$ Department of Obstetrics and Gynecology, University Clinics of Schleswig Holstein, Kiel, \\ Germany \\ ${ }^{3}$ Emirates Hospital,Jumeirah, Dubai, UAE \\ ${ }^{4}$ Morpheus Fertility Center, Pune, India
}

\section{Introduction}

Advancements in modern imaging techniques such as ultrasound, magnetic resonance imaging, computer tomography and other radiological procedures have improved the diagnosis of gynecological conditions to a great extent. However, the establishment of a final diagnosis and the initiation of appropriate treatment requires direct viewing of the uterine cavity as in hysteroscopy. In many cases, the patient can be treated during the initial hysteroscopy.

\section{Techniques and Patients}

One hundred and twenty-seven diagnoses established by transvaginal ultrasound and the corresponding outcomes of hysteroscopy and treatment were evaluated at the German Medical Center in Dubai Health Care City, UAE.

\section{Vaginal ultrasound, Saline infusion sonohysterography and Hysterosalpingo-contrast sonography}

We used the Samsung Accuvix A 30 ultrasound device with a 2D vaginal probe with enhanced directional power Doppler imaging (DPD) for the detection of peripheral blood vessels. Ambiguous intracavitary images of polyps, fibroids or adhesions were further clarified by using saline infusion sonohysterography (SIS) or hysterosalpingo-foam sonography (HyFoSy), which is a modification of hysterosalpingo-contrast sonography (HyCoSy). Saline infusion sonohysterography (SIS) is a valuable imaging tool to assess the uterus, the endometrial lining, and the endocervical canal. It is useful for evaluating a wide range of pathologies including polyps, leiomyomata, adhesions, anomalies, and endometrial hyperplasia or cancer [1]. SIS is a simple, inexpensive, and low-risk procedure; its findings complement those of a hysterosalpingogram (HSG), hystero-contrast sonography, or hysteroscopy. A 3D vaginal probe was not always at our disposal at the time.

\section{Diagnostic hysteroscopy, mini-hysteroscopy or office hysteroscopy}

Rigid hysteroscopes with an optical angle of 30 degrees (Figure 1) and flexible hysteroscopes (Figure 2) are available in the market. The published literature does not specify the size limits for mini-hysteroscopes. A mini-hysteroscope with a diameter of less than $5 \mathrm{~mm}$ can be introduced through the cervical canal without analgesia or dilatation. The majority of studies on mini hysteroscopy are focused on this category of scopes. Some mini-hysteroscopes are oval in cross-section, as is the internal os, and measure only $2.8 \mathrm{~mm}$ in diameter. A patient with a cervical stenosis or one who experiences severe pain during passage of the cervical canal might need a cervical block. Most mini-hysteroscopes have a working channel of 5-7 French for the insertion of graspers, scissors, polyp snares, or electrodes [2,3]. Valves for distension fluid are available in different shapes; a continuous flow system is recommended. Recent technological advancements favor thinner and smaller resectoscopes. The availability of small loops and a variety of working electrodes has made it possible to perform the mini-hysteroscopic procedure along with resection. Monopolar and bipolar electrodes are 
available. The bipolar electrode is recommended because the use of pure saline as distension medium is safest and minimizes the risk of water intoxication. Especially endometrial sampling in postmenopausal women with bleeding can be performed with greater sensitivity and specificity when the biopsy is performed under constant viewing. The cervix is entered either directly with the aid of a speculum or is inspected under vision during vaginoscopy. The hysteroscope is inserted in the cervical canal under continuous fluid pressure. Examination of, and any surgical procedures in, the uterine cavity require distension of the cavity by a continuous flow of fluid; the commonly used fluid is sterile saline. An angulated (30 degrees) optical device permits thorough screening of the uterine cavity. The continuous flow of fluid ensures clear vision and flushes away mucus, blood, and tissue debris. The cavity usually distends at a fluid pressure of 50-80 mmHg. The lowest pressure that provides sufficient distension is recommended. Excessively high pressure causes contraction of the uterus (pain) and loss of fluid into the abdominal cavity through the tubes. Sometimes a pressure of $125 \mathrm{mmHg}$ is needed for clear vision. A hysteroscopic pump may be used to maintain sufficient pressure when performing an office hysteroscopy. A pressure bag to compress a 1-liter bag of saline is not optimal but may help to perform the diagnostic hysteroscopy and biopsy when no hysteroscopic pump is available. This method requires a nurse to attend to the pressure bag and keep its pressure constant throughout the procedure. Bipolar electrodes for cutting and tissue evaporation require a conductive ion or electrolyterich medium to close the circuit between the active and the return electrode.

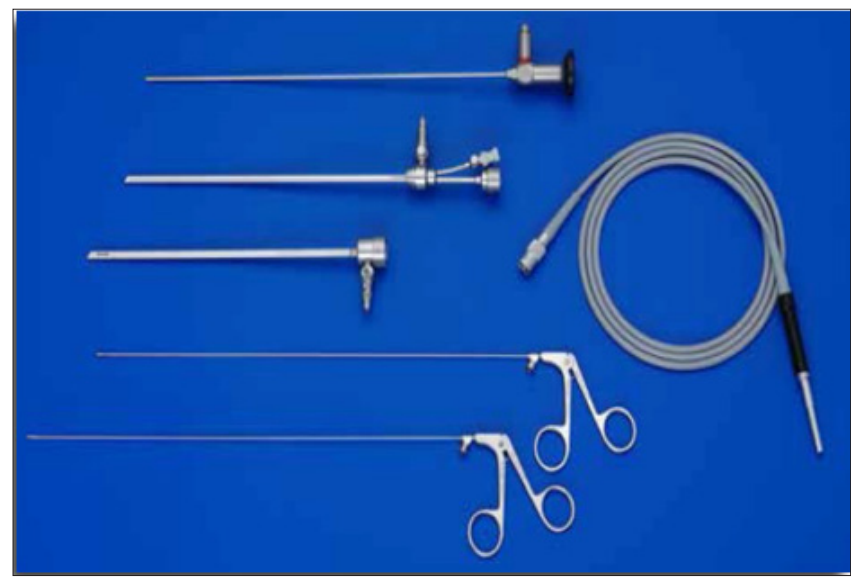

Figure 1: Rigid diagnostic hysteroscope with an operating channel
a. Oval shape
b. $5 \mathrm{~mm}$ diameter
c. $2 \mathrm{~mm}$ rod lens
d. 5-French channel
e. Inner and outer sheath
f. Semi-rigid instruments.

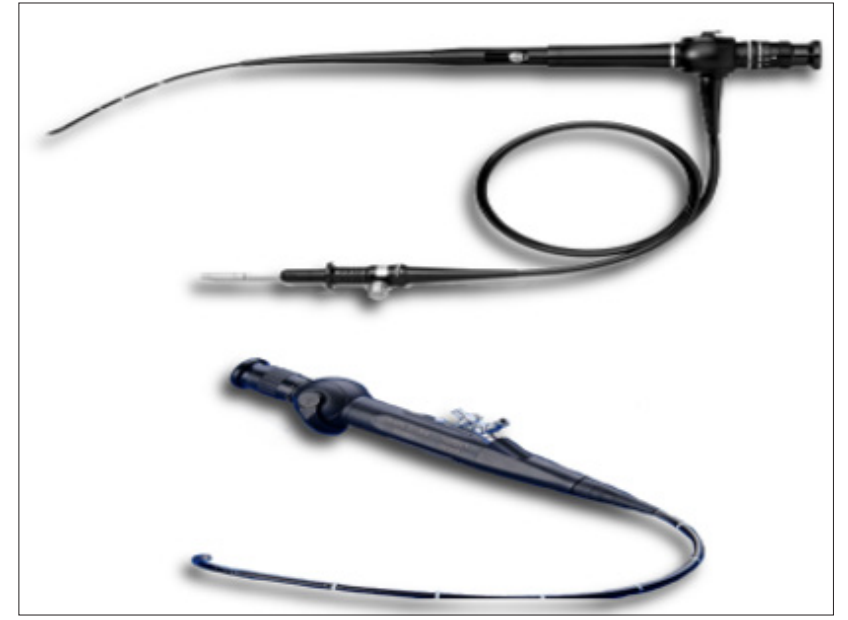

Figure 2: Flexible hysteroscope

1. $3-5 \mathrm{~mm}$ in diameter

2. Steerable tip

3. 5 to 7 French channel

4. Not ideal for surgical interventions.

In patients being treated for infertility, an office hysteroscopy is recommended to rule out intracavitary lesions prior to embryo transfer, and to treat small lesions through the operating channel of the diagnostic hysteroscope. When the investigation reveals a pathology that cannot be eliminated during the diagnostic or office hysteroscopy, or the patient has pain, she should be advised to undergo an operative hysteroscopy. Normally the cervix is not clamped. A slightly filled bladder helps to elevate the uterus into a stretched position. A useful office hysteroscope is ENDOSEE, along with its various modifications [4]; the device is economical and requires very little equipment.

\section{Operative hysteroscopy $[5,6]$}

The basic equipment for an operative hysteroscopy consists of the following items:

A. Hysteroresectoscope with a matching electrode and a 12$15^{\circ}$ angulated optical device

B. Light source with cable

C. Hysteromat with inflow and outflow tubes

D. Distension medium

E. Video camera

F. High-frequency unit for monopolar and bipolar current

The bipolar technique has extended the scope of operative hysteroscopy and made the procedure even safer. The decisive advantage of a bipolar hysteroscopy is that it can be performed without an electrolyte-free solution (sorbitol-mannitol solution). Instead, Ringer's or saline solution can be used; this reduces the cost of the distension medium. Bipolar hysteroscopy is currently the method of choice for high-risk situations (such as myomas grade 2/ESGE), 
because it is associated with a low rate of complications. Bipolar techniques are gaining increasing importance in operative hysteroscopy. Bipolar techniques in hysteroscopy help to solve some problems in intrauterine surgery and provide greater safety for the patient. Preferred indications for the bipolar surgical technique are myomas grade $2 /$ ESGE and large myomas $(>4 \mathrm{~cm})$. The main advantages of bipolar myoma resection include avoidance of the TUR syndrome (electrolyte shifts), and second or third surgeries as a result thereof. Fluid overload may occur even with the use of Ringer's solution. Care should be taken to ensure that the fluid deficit does not exceed three liters. Even greater caution is advised in anesthesiological risk groups. Besides using electrosurgery for simple mechanical destruction of tissue in fibroids and polyps, scissors or shavers with mechanical cutting and integrated suction systems are available. The Bigatti shaver (Storz) $[7,8]$ and the Truclear tissue removal system (Medtronic) are widely accepted options.

\section{Technique and procedure of operative hysteroscopy}

A. Diagnostic hysteroscopy with a $5 \mathrm{~mm}$ hysteroscope

B. Dilate the cervical canal to Hegar 7-9 (when using a smaller resectoscope for fertility surgery, only to Hegar 7)

C. Insert the outer sheath with an obturator

D. Replace the obturator with a hysteroresectoscope and matching electrode, and $12^{\circ}$ angulated lenses

E. Check the connections

i. $\quad$ Check the correct distension medium (bipolar: Ringer's solution; monopolar: sorbitol-mannitol solution)

ii. Inflow tube for the distension medium-airless and connected with the Hysteromat (irrigate beforehand!)

iii. Check pressure and flow selection on the Hysteromat

iv. Outlet tube in the collecting container

v. Connect with the electrosurgical unit and check the power level on the high-frequency unit (preselected programs for monopolar and bipolar surgery)
F. Open the inflow tube and perform distension of the uterine cavity. Irrigation is usually needed as the first step because dilatation of the cervix causes bleeding in the uterine cavity. Clear vision and adequate distension must be achieved before the procedure is started.

General and established indications for operative hysteroscopy are:
A. Infertility and abnormal bleeding
B. Septum dissection
C. Intrauterine adhesiolysis (grades III-IV/ESGE)
D. Myoma resection in one or a few submucous fibroids
E. Polyp resection
F. Endometrial ablation/endometrial resection
G. Resection of residual placenta

H. Opening or coagulation of hematometra in the rudimentary uterine horn

I. Removal of a lost IUD in the uterine cavity

Relative indications for operative hysteroscopy are the following:

A. Septum dissection with primary and secondary infertility

B. Resection of multiple myomas

\section{Our Experience}

At the German Medical Center in Dubai Health Care City, in an office setting without the equipment of an operating room, we encountered the following uterine pathologies over a period of two years (2017 to 2019) after performing palpation, vaginal ultrasound examinations, and HyFoSy (Table 1). One hundred and twentyseven patients were treated either directly through the operating channel of the diagnostic hysteroscope under conscious sedation or underwent an operative hysteroscopy in general anesthesia.

Table 1: Summarizes the new augmented reality techniques used to visualize the uterine cavity.

\begin{tabular}{|c|c|c|c|}
\hline \multicolumn{4}{|c|}{ New Augmented Reality Techniques to Explore the Uterine Cavity } \\
\hline & 1. Vaginal Ultrasound & 2. Diagnostic Hysteroscopy & 3. Operative Hysteroscopy \\
\hline & $\begin{array}{l}\text { Saline infusion sonography and hysterosal- } \\
\text { pingo contrast sonography }\end{array}$ & $\begin{array}{l}\text { Mini hysteroscopy or office } \\
\text { hysteroscopy }\end{array}$ & $\begin{array}{l}\text { The necessary basic equipment for operative hysterosco- } \\
\text { py consists of the following items }\end{array}$ \\
\hline $\begin{array}{l}\text { Instruments } \\
\text { and Tech- } \\
\text { niques }\end{array}$ & $\begin{array}{c}\text { Samsung Accuvix A } 30 \text { sonographic machine } \\
\text { with a 2D vaginal probe with enhanced } \\
\text { Directional power Doppler Imaging (DPD)/ } \\
\text { Saline Infusion Sonohysterography (SIS) } \\
\text { or by hysterosalpingo foam sonography } \\
\text { (HyFoSy) }\end{array}$ & $\begin{array}{l}\text { Rigid hysteroscopes with an } \\
\text { optical angle of } 30 \text { degrees } \\
\text { (Figure 1) and flexible hys- } \\
\text { teroscopes (Figure 2) }\end{array}$ & $\begin{array}{l}\text { Hysteroresectoscope with matching electrode and } \\
\qquad 120-150 \text { angled optic with } \\
\text { Light source with cable } \\
\text { a. Hysteromat with inflow and outflow tube } \\
\text { b. Distending Medium } \\
\text { c. Video camera } \\
\text { d. High-frequency unit for monopolar and bipolar current }\end{array}$ \\
\hline
\end{tabular}


In 19 patients the thickened endometrium could be treated by performing a biopsy and/or curettage (Figure 3). In six patients we performed endometrial ablation with a bipolar loop and a roller ball. In 42 patients with endometrial polyps and 35 with submucosal fibroids, the imaging investigation was complemented with saline infusion ultrasonography. The patients then underwent an operative hysteroscopy, during which polyps and fibroids were resected under vision, followed by dilation and curettage. No unexpected malignancy was encountered. In three further patients we suspected an intrauterine malignancy and performed an endometrial biopsy under vision solely for diagnostic purposes. In 18 patients undergoing treatment for infertility, the hysteroscopy was performed together with a laparoscopy; no pathologies were suspected. A uterine septum or a fundal cleft was resected in seven women. Four of these women only had a fundal cleft, but the latter are known to hinder implantation. In four patients, a preoperatively diagnosed septum was divided with the hook electrode during the operative hysteroscopy. The operative procedures performed in the 127 patients are summarized in Table 2.

Table 2: 127 operative hysteroscopic procedures.

\begin{tabular}{|c|c|}
\hline Hysteroscopy with endometrial biopsies & $\mathbf{n}=\mathbf{1 9}$ \\
\hline Hysteroscopy and endometrial ablation & $\mathrm{n}=6$ \\
\hline Hysteroscopic polypectomies & $\mathrm{n}=42$ \\
\hline Hysteroscopic myomectomies & $\mathrm{n}=35$ \\
\hline $\begin{array}{c}\text { Endometrial biopsy for a suspected malignancy } \\
\text { which revealed 7 septa not detected preoperatively. }\end{array}$ & $\mathrm{n}=3$ \\
\hline Primarily diagnosed uterine septa & $\mathrm{n}=4$ \\
\hline Total & $\mathrm{n}=127$ \\
\hline
\end{tabular}

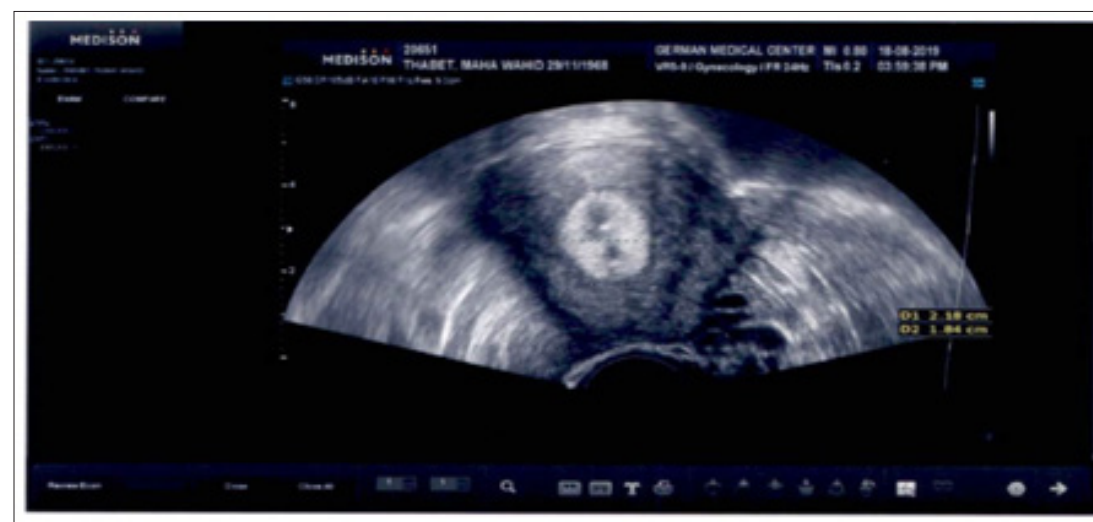

(a)

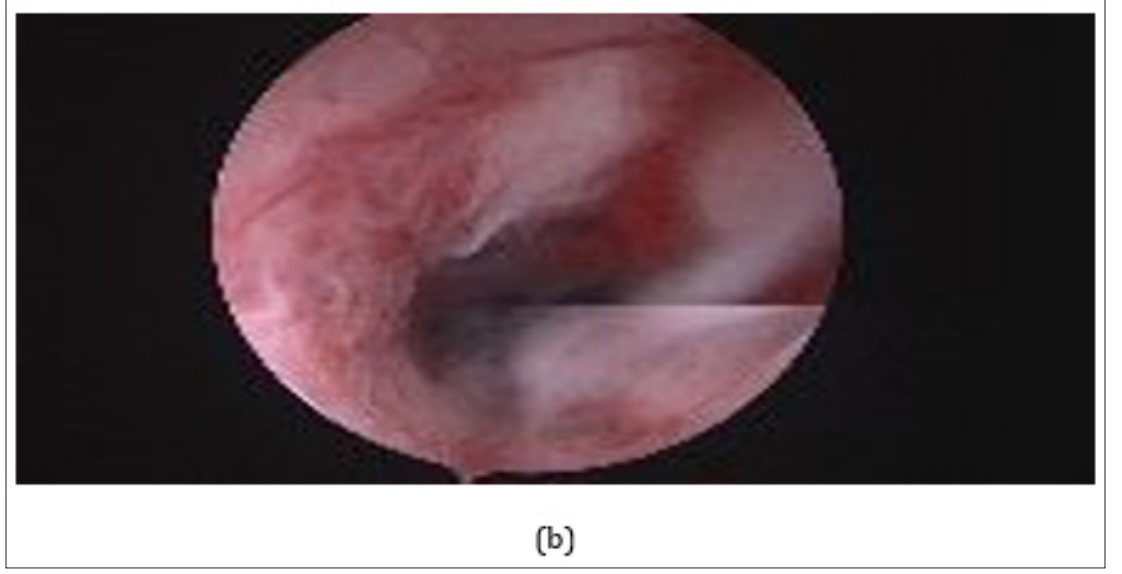

Figure 3: Vaginal ultrasound

a. Compared to hysteroscopy

b. In a 49-year-old patient with endometrial hyperplasia.

One hundred and twenty-six operative procedures were performed without complications. One patient underwent submucous bipolar resection of a myoma measuring $4 \mathrm{~cm}$ in size and experienced a pulmonary embolism after 24 hours. Regrettably, the patient was not given heparin. She reported respiratory problems on the phone and underwent an immediate control investigation which revealed her condition. The patient was treated accordingly and recovered well. 


\section{Discussion, Future Perspectives, Conclusion}

Barring pregnancies, which are the domain of fetal medicine, the uterine cavity has ceased to be a mystery. Ultrasound imaging in conjunction with SIS and HyFoSy are valuable techniques for diagnostic purposes. They can be used for performing an office hysteroscopy. Minor surgical procedures can also be performed in this setting through the operating channel of the diagnostic hysteroscope. Alternatively, the surgeon may perform an operative hysteroscopy in general anesthesia. All of our patients underwent their preliminary examinations in the office without analgesia or anesthesia. Many lesions were clearly seen on two-dimensional ultrasound (Figure 4) conditions such as septa were seen even more clearly on three-dimensional ultrasound (Figure 5a\&5b).

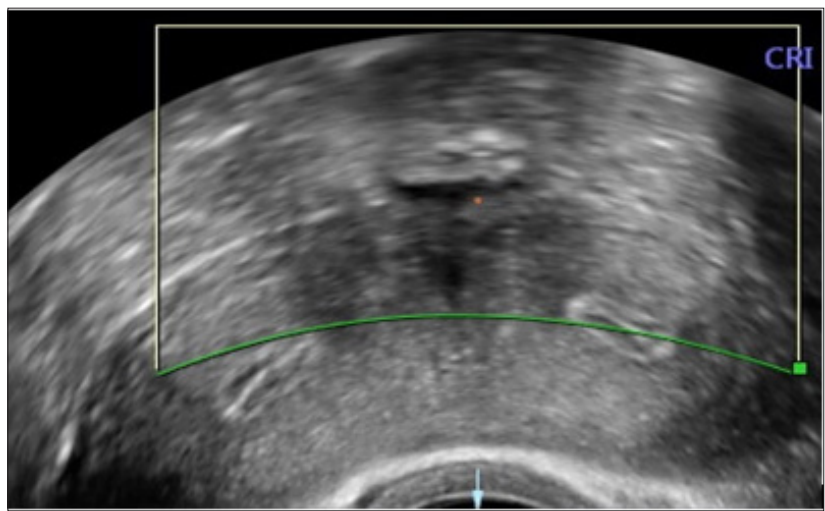

Figure 4: Imaging of the septate uterus. 2D ultrasound with two separate endometrial echoes in the transverse plane.

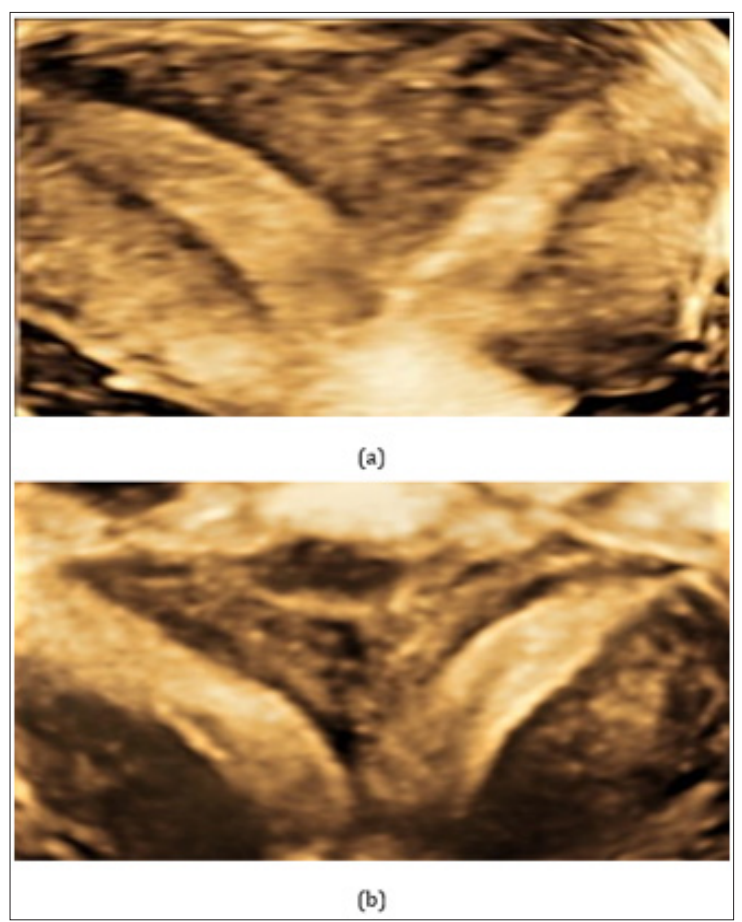

Figure 5: Image of a septate uterus (a)\&(b) using three-dimensional transvaginal ultrasonography (3D).
In our work environment in the UAE, minor surgical procedures for diagnostic purposes as well as operative hysteroscopies are performed in a day-care setting, under conscious sedation or anesthesia. Office hysteroscopies performed for diagnostic purposes may also include extensive work through the operating channel of the hysteroscope by the use of graspers, scissors, or catheters for canalization of the proximal tube. Figure 6 shows a patient with a suspected septum who underwent a hysteroscopy. Figure 7 shows the two-dimensional ultrasound image and the corresponding hysteroscopic finding of a submucous fibroid.

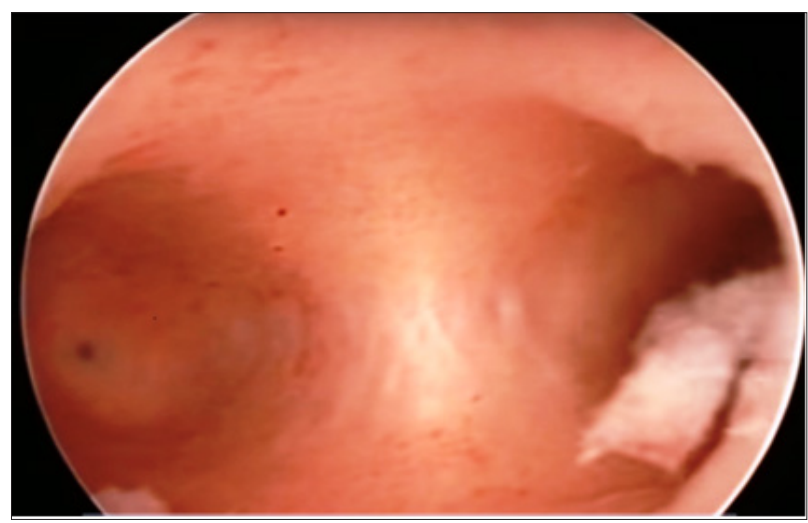

Figure 6: A septate uterus on hysteroscopy.

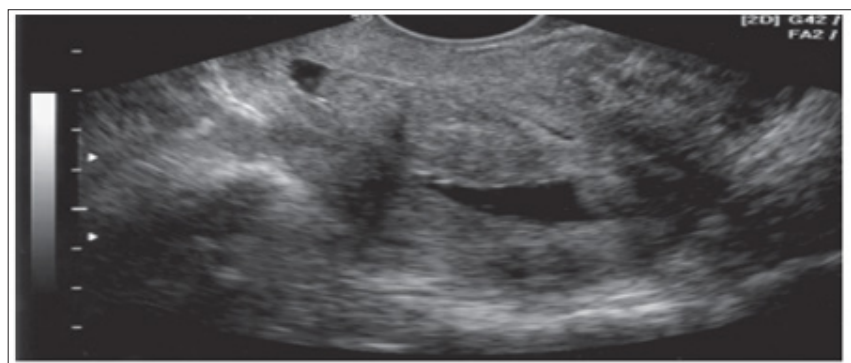

(a)

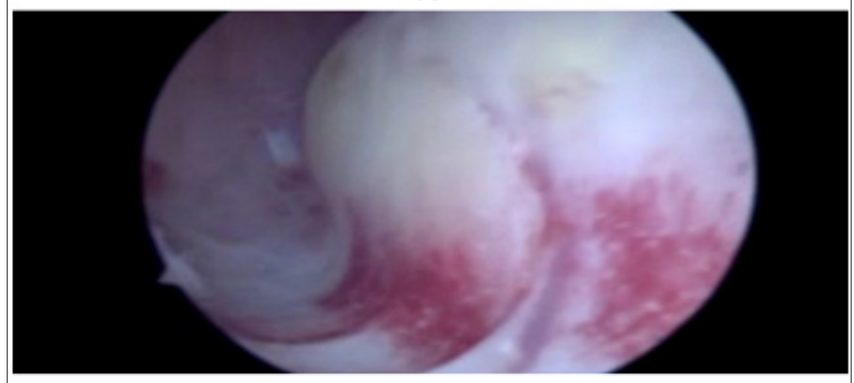

(b)

Figure 7: a. Submucous fibroid seen clearly on 2D ultrasound with saline infusion

b. Compared to its hysteroscopic presentation before enucleation.

\section{Summary}

The large majority of conditions in the uterus can be detected and treated, provided the patients consult their doctors at the very onset of symptoms and undergo regular annual or biannual 
gynecological controls with palpation and a vaginal ultrasound examination. On ultrasound, the uterine cavity is visualized better by the transvaginal approach than through the abdomen. Hysteroscopy is one of the many procedures used in minimally invasive surgery (MIS), frequently in the postoperative setting, and has expanded the scope of surgical gynecology. Operative hysteroscopy has become a standard gynecological procedure and should be a part of the surgical repertoire at every hospital. Future developments include new and smaller scopes, possibly the revival of flexible scopes, novel concepts of modular digital hysteroscopy, instruments that provide better vision, and simplified all-in-one instruments. Nanorobotics may pave the way for the creation of the "hysterorobot".

\section{References}

1. Debra L, Thomas C (2004) Saline infusion sonohysterography: Technique, indications, and imaging findings. J Ultrasound Med 23(1): 97-112.

2. Bettocchi S, Achilarre MT, Ceci O, Luigi S (2011) Fertility-enhancing hysteroscopic surgery. Semin Reprod Med 29(2): 75-82.
3. El Toukhy T, Campo R, Khalaf Y, Tabanelli C, Gianaroli L, et al. (2016) Hysteroscopy in recurrent in-vitro fertilization failure (TROPHY): A multicentre, randomised controlled trial. Lancet 387(10038): 26142621.

4. Indman Paul (2011) New device makes office-based hysteroscopy possible. Laparoscopy and Hysteroscopy, Contraception, American Association of Gynecologic Laparoscopists, USA.

5. (2013) Practical Manual for laparoscopic and hsteroscopic gynecological surgery ( $2^{\text {nd }}$ edn), In: Schollmeyer T, Mettler L, Rüther D, Alkatout I (Eds.), Jaypee Brothers Medical Publishers Ltd., New Delhi, India.

6. Alkatout I, Mettler L (2019) Practical Manual for laparoscopic and hysteroscopi gynecological surgeries, Kiel school of gynecological endoscopy ( $3^{\text {rd }}$ edn), Jaypee Brothers Medical Publishers, New Delhi, India.

7. Bigatti G (2011) IBS® integrated bigatti shaver, an alternative approach to operative hysteroscopy. Gynecol Surg 8(2): 187-191.

8. Bigatti G, Ferrario C, Rosales M, Baglioni A, Bianchi S (2012) IBS $®$ integrated bigatti shaver versus conventional bipolar resectoscopy: A randomized comparative study. Gynecol Surg 9: 63-72. 